\title{
MATLAB Package and Science Subjects for Undergraduate Studies
}

\author{
Hazim H. Tahir ${ }^{1}$, Teresa Fernández Pareja ${ }^{2}$ \\ Ministry of Science and Technology, Baghdad, Iraq $^{1}$ \\ Universidad Politécnica de Madrid, Spain ${ }^{2}$
}

\begin{abstract}
Research study to elicit and design a simplified method in order to model the scientific concepts and basics for many learning fields and disciplines such as Mathematics, Physics, Chemistry, Statistics, Electronics, Communications, Systems \& Control, etc using the MATLAB package that has been taught in Universities and in High Schools to establish a new generation of graduates by using scientific and modern academic curricula. First year Mathematics was taken as an example subject in this study and could be generalized to the other subjects. Graphical user interfaces (GUI) program developed and implemented to solve almost all the problems that first year Mathematics curriculum contains. This proposal is cost effective and its plan is technically feasible since the fulfillment of this goal would revolutionize teaching and education, and it will make it possible for many more students to learn, do, and appreciate MATLAB programming, since $M A T L A B$ would build the skills that are necessary to compete for the jobs of tomorrow.
\end{abstract}

\section{Introduction}

This proposal will try to persuade The Ministry of Education / Science \& Technology to develop and introduce special curriculum content for teaching college and high school students the MATLAB package to help them tackle and solve problems. Since problem solving is identified as a top priority in many curricula in science, technology and engineering.

MATLAB (short for MATrix LABoratory) is a high-performance interacting data-intensive software environment for high-efficiency engineering and scientific numerical calculations [1, 4, 8, 11, and 17]. Applications include: heterogeneous simulations and data-intensive analysis of very complex systems and signals, comprehensive matrix and arrays manipulations in numerical analysis, finding roots of polynomials, and two or three-dimensional plotting and graphics for different coordinate systems, integration and differentiation, signal processing, control, identification, symbolic calculus, optimization, etc $[1,6,20]$. The goal of MATLAB is to enable the users to solve a wide spectrum of analytical and numerical problems using matrixbased methods [5], attain excellent interfacing and interactive capabilities, compile with high-level programming languages, ensure robustness in dataintensive analysis and heterogeneous simulations, provide easy access to and straightforward implementation of state-of-the-art numerical algorithms, guarantee powerful graphical features, etc [11]. Due to high flexibility and versatility, the MATLAB environment has been significantly enhanced and developed during recent years. This provides users with advanced cutting-edge algorithms, enormous data-handling abilities, and powerful programming tools [7].

MATLAB is based on a high-level matrix / array language with control flow statements, functions, data structures, input / output, and object-oriented programming features [6].

MATLAB was originally created to provide easy access to matrix software developed by the LINPACK and EISPACK matrix computation software. A numerical analyst called Cleve Moler wrote the first version of MATLAB in the 1970s [7, 19]. It has since evolved into a successful commercial software package. MATLAB, also, has progressed over the last 20 years and became the standard instructional tool for introductory and advanced courses in science, engineering, and technology. The MATLAB environment allows one to integrate user-friendly tools with superior computational capabilities. Users can practice and appreciate the MATLAB environment interactively, enjoying the flexibility and completeness, analyzing and verifying the results by applying the range of build-in commands and functions, and expanding MATLAB by developing their own applicationspecific files, etc $[10,17]$. Users quickly access data files, programs, and graphics using MATLAB help. A family of application-specific toolboxes, with a specialized collection of $\mathrm{m}$-files for solving problems commonly encountered in practice ensures comprehensiveness and effectiveness. SIMULINK is a companion graphical mouse-driven interactive environment enhancing MATLAB [2, 13]. SIMULINK $^{\circledR}$ is used for simulating linear and nonlinear continuous-time and discrete-time dynamic systems. The MATLAB features are illustrated in Figure 1. 


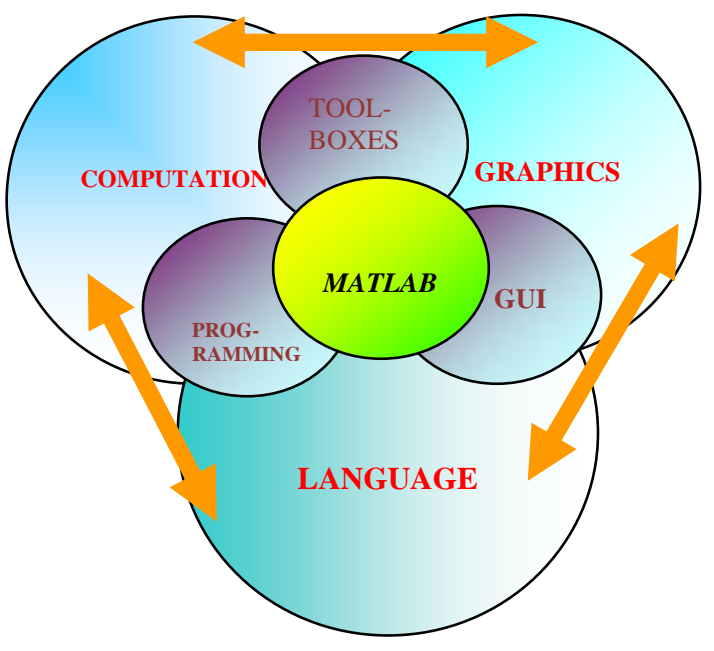

Figure 1. MATLAB features

In this proposal, the need for MATLAB as a pedagogical tool in Science / Engineering is highlighted. The proposal concludes with relevant observations and recommendations.

A First year university Mathematics course was taken as an example. A graphical user interfaces (GUI) program was developed and implemented to help students solve their mathematical tutorials / problems.

\section{What is MATLAB?}

MATLAB is a high-performance language for technical computing. It integrates computation, visualization, and programming in an easy-to-use environment where problems and solutions are expressed in familiar mathematical notation [3, 4, and 6]. Typical uses include:

- Math and computation

- Algorithm development

- Modeling, simulation, and prototyping

- Data analysis, exploration, and visualization

- Scientific and engineering graphics

- Application development, including graphical user interface building

MATLAB is an interactive system whose basic data element is an array that does not require dimensioning. This allows the user to solve many technical computing problems, especially those with matrix and vector formulations, in a fraction of the time it would take to write a program in a scalar non-interactive language such as $\mathrm{C}$ or FORTRAN.

MATLAB has developed over a period of years with input from many users. In university environments, it is the standard instructional tool for introductory and advanced courses in mathematics, science and engineering. In industry, MATLAB is the tool of choice for highproductivity research, development, and analysis [1, 12, 15 and 16].

\subsection{The MATLAB System}

The MATLAB environment features a family of application-specific toolboxes that integrate specialized m-files that extend MATLAB in order to approach and solve particular application-specific problems [13, 18]. MATLAB system environment consists of five main parts:

- The MATLAB language. This is a high-level matrix/array language with control flow statements, functions, data structures, input/output, and object-oriented programming features. It allows both "programming in the small" to rapidly create quick and dirty throwaway programs, and "programming in the large" to create complete large and complex application programs [6, 7, 18 and 19].

- Development Environment. This is the set of tools and facilities that help the user to use MATLAB functions and files. Many of these tools are graphical user interfaces. It includes the MATLAB desktop and Command Window, a command history, and browsers for viewing help, the workspace, files, and the search path [6, 7, and 19].

- Handle Graphics ${ }^{\circledR}$. This is the MATLAB graphics system. It includes high-level commands for two-dimensional and three-dimensional data visualization, image processing, animation, and presentation graphics. It also includes low-level commands that allow the user to fully customize the appearance of graphics as well as to build complete graphical user interfaces (GUI's) on their MATLAB applications [9, 11].

- The MATLAB Mathematical Function Library. This is a vast collection of computational algorithms ranging from elementary functions like sum, sine, cosine, and complex arithmetic, to more sophisticated functions like matrix inverse, matrix Eigenvalues, Bessel functions, and fast Fourier transforms [1618].

- The MATLAB Application Program Interface (API). This is a library that allows the user to write $\mathrm{C}$ and FORTRAN programs that interact with MATLAB. It include facilities for calling routines from MATLAB (dynamic linking), calling MATLAB as a computational engine, and for reading and writing MAT-files [6, 15, 18, 19].

\subsection{What is Simulink?}

Simulink, a companion program to MATLAB, is an interactive system for simulating nonlinear dynamic systems. It is a graphical mouse-driven program that allows the user to model a system by drawing a block diagram on the screen and 
manipulating it dynamically. It can work with linear, nonlinear, continuous-time, discrete-time, multi-rate, and hybrid systems [2, 13].

Blocksets are add-ons to Simulink that provide additional libraries of blocks for specialized applications like communications, signal processing, and power systems $[2,11,13]$.

\section{MATLAB and Its Toolboxes}

MATLAB distributed by The Mathworks, is a technical computing environment for high performance numeric computation and visualization. It integrates numerical analysis, matrix computation, signal processing, and graphics in an easy-to-use environment [11].

MATLAB also, features a family of applicationspecific solutions called toolboxes. They are very important to most users of MATLAB, toolboxes allow users to learn and apply specialized technology. Toolboxes are comprehensive collections of MATLAB functions (m-files) that extend its environment in order to solve particular types of problems [5, 6, 17]. The Table 1 below includes the toolboxes that are available in the latest version of MATLAB

Table 1. MATLAB toolboxes

\begin{tabular}{|c|c|c|}
\hline Communications & Image Processing & System Identification \\
\hline Control System & Instrument Control & Wavelet \\
\hline Data Acquisition & Mapping & MATLAB Compiler \\
\hline Database & Neural Network & $\begin{array}{c}\text { MATLAB C/C++ Graphics } \\
\text { Library }\end{array}$ \\
\hline Datafeed & Optimization & MATLAB C/C++ Math Library \\
\hline Filter Design & Partial Differential Equation & MATLAB Report Generator \\
\hline Financial & Robust Control & MATLAB Runtime Server \\
\hline $\begin{array}{c}\text { Frequency Domain System } \\
\text { Identification }\end{array}$ & Signal Processing & MATLAB Web Server \\
\hline Fuzzy Logic & Statistics & Simulink \\
\hline $\begin{array}{c}\text { Higher-Order Spectral } \\
\text { Analysis }\end{array}$ & Spline & Symbolic/Extended Math \\
\hline
\end{tabular}

\section{What MATLAB can do and tackle?}

This will provide the reasoning for MATLAB applications. MATLAB can solve from easy to advanced application-specific problems to illustrate the applicability and versatility of the MATLAB environment $[15,20]$. For example, in a multivariable calculus subject, students can study and perform the following using MATLAB programming: parametric and polar equations, vectors, coordinate systems (Cartesian, cylindrical, and spherical), vector-valued functions, derivatives, partial derivatives, directional derivatives, gradient, optimization problems, multiple integration, integration in vector fields, and other topics. In contrast, linear algebra emphasizes matrix techniques for solving systems of linear and nonlinear equations covering matrices and operations with matrices, determinants, vector spaces, independent and dependent sets of vectors, bases for vector spaces, linear transformations, Eigenvalues and Eigenvectors, orthogonal sets, least squares approximation, interpolation, etc [3,4,6-8]. The MATLAB environment is uniquely suitable to solving such a variety of problems in science and engineering. Using the calculus and physics background, a variety of real-world scientific / engineering problems can be attacked and resolved.

MATLAB integrates computation, visualization, and programming in an easy-to-use systematic, robust and computationally efficient environment where problems and solutions are expressed in familiar (commonly used) mathematical notation [12, 14, 15]. The user can perform mathematic computation, algorithm development, simulation, prototyping, data analysis, visualization, interactive graphics, and application-specific developments including graphical user interface (GUI) features by using just one or two commands [ 9, 11-13, 16, 21]. In MATLAB the data is manipulated in the array form, allowing the user to solve complex problems. It was emphasized that the MATLAB environment was originally developed using data-intensive matrix computation methods.

\section{Advantages of MATLAB}

MATLAB offers a number of significant advantages over traditional high-level programming languages such as FORTRAN or C:

- More power. Using MATLAB, a student or engineer can solve difficult problems in less time and with less effort [1, 3-5, 8, 10, 20].

- Greater ease of use. MATLAB is easier to master than a high-level programming language. As a result, the students have the tools to solve significant engineering problems much earlier in the semester [4, 6, 8].

- Emphasis on problem solving. Rather than becoming entangled in language issues, students can concentrate fuller on the problem to be solved. Students can tackle more problems of greater complexity and still have time to analyze their results $[1,4,5,10,18,20]$.

- Fewer errors. When using MATLAB, students make fewer errors. Moreover, they are more able to find and correct the errors themselves, requiring less help from instructors and teaching assistants [3, 13, 17, 18]. These advantages provide only a brief glimpse of the power and flexibility of the MATLAB system $[7,18,19]$.

\section{Developing Matlab GUI's Program}

MATLAB Version 5 and beyond allows the user to build GUIs (or graphical user interfaces). A GUI 
is simply an interface that can call upon different MATLAB operations and perform them without the user needing to know the MATLAB language. In MATLAB, typing the command for the GUI creates a window where graphs, text boxes and clickable buttons appear that carry out a series of MATLAB commands [16]. The GUI's programs (MATH 1 \& MATH 2) can be seen in Figure 2 and Figure 3 respectively. In Figure 2 the following calculations could be done: Determinant, Eigenvalues, Eigenvectors, Inverse and Transpose of a Matrix. In Figure 3 the following calculations could be done: Roots of the polynomial, Differentiation, Integration, Curve Drawing, Laplace Transform, and Inverse Laplace Transform.

These programs will inspire, motivate and help students relate in-class mathematical concepts to real-world events. It would make them feel tremendous satisfaction after checking and comparing their manual solutions of the problems to those obtained by using these GUI's programs [20, 21].

These two programs will also cultivate interest, raise achievement and will build the skills that students need to compete for tomorrow's jobs.

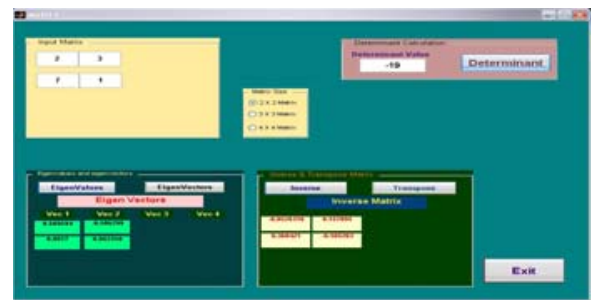

Figure 2. Calculations of the following: determinant, eigenvalues, eigenvectors, inverse and transpose of a matrix

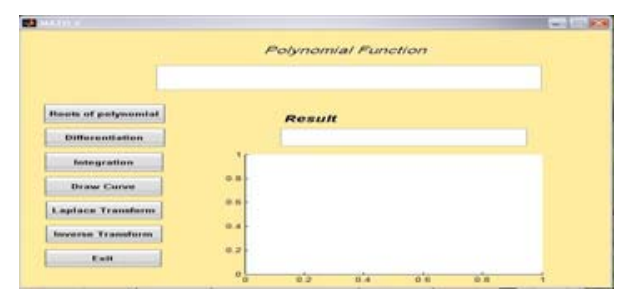

Figure 3. Calculations of the following: Roots of the polynomial, Differentiation, Integration, Curve Drawing, Laplace Transform, and Inverse Laplace Transform

\section{Integrating MATLAB into Classical First Year Mathematical Curriculum}

The simple assumption of "integrating MATLAB into a mathematics curriculum" is not at all simple to students. Since the curriculum was chosen and cannot be altered, a supplemental booklet could be printed and issued by the Department to help students and roaming tutors to work and use MATLAB. The supplemental booklet will contain full explanations of the GUI programs (MATH 1 \&
MATH 2) that would be used in the computer/tutorial sessions. This booklet consists of all the tutorial questions of each chapter of the curriculum that students should solve and check during their computer sessions, students will then discover the correct solutions, i.e. this booklet should link with the lectures material.

\section{Conclusions}

In this proposal, the need for MATLAB as a pedagogical tool in Science / Engineering is highlighted. The proposal concludes with relevant observations and recommendations.

The MATLAB package is very powerful and much easier and quicker to master than any other traditional programming language $(\mathrm{C}++$, Visual basic etc). This package will inspire and motivate students, and will help them relate in-class mathematical concepts to real-world events and will make them feel tremendous subsequent satisfaction. This, also, will broaden science, technology and engineering education.

The potential benefits of teaching MATLAB are immeasurable, since it is widely used in classes and is proving to be a very effective teaching aid.

One proposal is that The Ministry of Education/ Science \& Technology should make the development of teaching MATLAB its foremost goal and should seek support for the pursuit of this goal from research and education communities. This proposal is cost effective and its plan is technically feasible since the fulfillment of this goal would revolutionize teaching and education. It will also make it possible for many more students to learn, practice, and appreciate MATLAB programming, since MATLAB will build the skills that are necessary to compete for tomorrow's jobs.

\section{Recommendations}

The following recommendations are made for the use of MATLAB as a pedagogical tool in science, technology and engineering education:

- MATLAB should be introduced in the nation's science and engineering faculties and made compulsory for all science and engineering students.

- Science and engineering faculties should encourage their teaching staff to attend workshops/seminars on the most recent version of MATLAB.

- MATLAB groups among students should be encouraged in all the nation's universities. These groups should be involved in discussions about problems encountered and successes made whilst solving a particular problem. 
- Calculations and analysis done using MATLAB should be well documented for future references.

- Inter-university and inter-departmental competitions among students on MATLAB and its programming application should be encouraged to stimulate interest in the use of the software.

The Ministry of Education / Science \& Technology must ensure that the introduction and use of MATLAB is compulsory throughout the nation's science and engineering faculties and this can be achieved through the introduction of appropriate legislation.

\section{References}

[1] Biran, A. and Breiner (1995). M. MATLAB for Engineers. Addison-Wesley.

[2] Dabney, J. B. and Harman, T. L. (1998). Mastering Simulink 2. Upper Saddle River, NJ: Prentice Hall.

[3] Eric Weisstein. World of Mathematics. Prime Spiral. http://mathworld.wolfram.com/PrimeSpiral. html. (AccessDate 17 Augest 2007).

[4] Etter, D.M. (1997). Engineering Problem Solving with $M A T L A B$. 2nd Edition, Prentice Hall.

[5] Gottling, J.G. (1995). Matrix Analysis of Circuits Using MATLAB. Prentice Hall.

[6] Hanselman, D. C. and Littlefield, B. (2001). Mastering MATLAB 6: A Comprehensive Tutorial And Reference. Upper Saddle River, NJ.: Prentice Hall.

[7] Higham, D.J. and Higham, N. J. (2000). MATLAB Guide. SIAM, Philadelphia.

[8] Lindfield, G.R., and Peny, J. E. T. (2000). Numerical Methods Using MATLAB. Upper Saddle River, NJ. : $8^{\text {th }}$ Edition, Prentice-Hall.

[9] Marchand, Patrick. (1999). Graphics and GUI's with $M A T L A B$. Second Edition, Boca Raton: CRC Press.

[10] M. Nagrial (2002). Education and training in engineering software and applications. Int. Conference on Engineering Education, www. citeseer.nj.nec.com/560624.html. (Access Date 11 Augest 2007).

[11] Nakamura, Shoichiro (2002). Numerical Analysis and Graphic Visualization with MATLAB. Upper Saddle River, NJ.: $2^{\text {nd }}$ Edition, Prentice-Hall

[12] Okoro, O. I., Govender, P. and Chikuni, E. (2006). A New User-Friendly Software for Teaching and Research in Engineering Education. Pacific Journal of Science and Technology, volume 7, Number 2.
[13] Okoro, O.I., (2005). Introduction to MATLAB/ SIMULINK for Engineers and Scientists. Enugu, Nigeria John Jacob’s Classic Publishers.

[14] Orsak, G. C. et al., (2005). Engineering Our Digital Future. Upper Saddle River, NJ.: Perason Prentice-Hall Inc.

[15] Palm, W. J. (2001). Introduction to MATLAB for Engineers. Boston, MA.: McGraw-Hill.

[16] Quinlan, J. E. (2004). Developing graphical interfaces for interactive applications in Matlab using GUIDE. MSc thesis, The Ohio State University, Department of Mathematics.

[17] Recktenwald G (2000). Numerical Methods with MATLAB: Implementations and Applications.Upper Saddle River, NJ.: Prentice Hall.

[18] Shampine, L.F., I. Gladwell, and S. Thompson (2003). Solving ODEs with MATLAB. New York: Cambridge University Press.

[19] Sigmon, K. and T.A. Davis (2002). MATLAB Primer. Sixth Edition. Chapman and Hall/CRC.

[20] User's Guide (1995). The Student Edition of MATLAB: The Ultimate Computing Environment for Technical Education. Mathworks, Inc., Upper Saddle River, NJ.: Prentice Hall.

[21] Tahir, Hazim H., Teresa Fernández Pareja (2007). Should undergraduate science / engineering Students be taught MATLAB package?. Primer Congreso Internacional de Matemáticas en Ingeniería y Arquitectura, PP.387-392, [30 May-1 June 2007], Madrid, Spain: Univesidad Politécnica de Madrid.

[22] Tonkes, E.J., Loch, B. I. and Stace, A. W (2005). An innovative learning model for computation in first year mathematics. International Journal of Mathematics Education in Science and Technology, 36 (7). PP. 751-759, ISSN 1464-5211. 